\title{
Symmetry, Statistics and Structure in MHD Turbulence
}

\author{
John V. Shebalin \\ NASA Johnson Space Center, Mail Code KA, Houston, TX 77058
}

\begin{abstract}
It is known that symmetries inherent in the statistical theory of ideal (i.e., nondissipative) magnetohydrodynamic (MHD) turbulence are broken dynamically to produce coherent structure. Previous numerical investigations are extended to study decaying MHD turbulence. Here, we find that coherent structure also arises and persists in the presence of dissipation. Thus, the magnetic dynamo may be due to a dynamically broken symmetry that is inherent to MHD turbulence.
\end{abstract}

Keywords: MHD, Turbulence, Statistical Mechanics, Magnetic Dynamo

PACS: 52.35.Ra, 52.65.-y, 91.25.Cw, 94.05.Lk, 96.50.Tf

\section{INTRODUCTION}

In this work, incompressible, homogeneous 3-D MHD turbulence is examined in terms of truncated Fourier models. Physical fields such as the turbulent magnetic induction $\mathbf{b}$ are represented by truncated Fourier series on an $N^{3}$ grid:

$$
\mathbf{b}(\mathbf{x})=\sum_{0<|\mathbf{k}| \leq K} \tilde{\mathbf{b}}(\mathbf{k}) e^{i \mathbf{k} \cdot \mathbf{x}} .
$$

The wave vectors $\mathbf{k}$ in $\mathrm{k}$-space have integer components between $-N / 2$ and $+N / 2$, while the corresponding discrete set of position vectors $\mathbf{x}$ in $\mathrm{x}$-space have components that are integer multiples of $2 \pi / N$, where $N$ is the number of grid points in each dimension. The 'isotropic truncation' radius is $K$, which is chosen to ensure that retained coefficients fit within a sphere in k-space; the exact value of $K$ is dictated by de-aliasing requirements [Patterson 1971].

These models place MHD turbulence in a periodic box that can represent both a small volume interior to a larger volume containing plasma, and also a complete mass of plasma within a 3-torus (such as the universe). Furthermore, isolated plasma (such as a star) may be thought of as residing in box large enough so that any plasma flows or magnetic fields outside of or on the surface of the box have negligible energy. For this later case, a Fourier model can serve as an approximate surrogate. Thus, Fourier models are of value by providing at least qualitative information about geophysical and astrophysical turbulence, especially if dissipation is included.

Statistical studies of such Fourier models were initiated by T. D. Lee, in a prescient paper [Lee 1952] that demonstrated the existence of a canonical ensembles based on the ideal invariants in the phase space defined by independent Fourier modes. The total energy $E$ is one ideal invariant, and two other invariants were soon discovered: magnetic helicity $H_{M}$ [Elsässer 1956] and cross helicity $H_{C}$ [Woltjer 1958]. Once computational resources were sufficient, the basic 'absolute equilibrium ensemble theory' 
was described for three-dimensional (3-D) MHD turbulence [Frisch 1975]. The broken symmetry and non-ergodicity contained in this theory was subsequently recognized [Shebalin 1982, 1983, 1989, 1994, 1996, 1998, 2002].

A recent paper [Shebalin 2006] examines ideal, homogeneous, incompressible, 3-D MHD turbulence in a rotating frame of reference with and without a mean magnetic field. There turn out to be five general cases of incompressible, homogeneous 3-D MHD turbulence, differentiated by the absence or presence of a mean magnetic field $\mathbf{B}_{0}$ and/or an overall system rotation $\Omega_{0}$; these cases are shown in Table 1 . There, a new invariant, the parallel helicity $H_{P} \equiv H_{C}-\sigma H_{M}$ is indicated, which appears when $\Omega_{\mathrm{o}}=\sigma \mathbf{B}_{\mathrm{o}} \neq 0$. (For further details, including details of very long-time $32^{3}$ simulations, please see [Shebalin 2006].)

Here, dissipation is introduced through non-zero viscosity $v$ in eq. (2) and magnetic diffusivity $\eta$ in eq. (3). Results from a long-time $64^{3}$ simulation are presented and largescale coherent structures, as seen in previous ideal runs, are still present. In effect, this is a 'magnetic dynamo process' that is inherent to MHD turbulence, a process that requires an initial energetic stirring of a magneto-fluid, but does not require any continuous external forcing, such as convection. A magnetic dynamo may thus be related more to an inherent, dynamically broken symmetry, than to external forcing.

\section{BASIC THEORY}

The well-known non-dimensional form of the MHD equations in a rotating frame of reference with constant angular velocity $\Omega_{\mathrm{o}}$ and a mean (i.e., uniform and constant) magnetic induction $\mathbf{B}_{\mathrm{o}}$ are (where $\nabla \cdot \mathbf{u}=\nabla \cdot \mathbf{b}=0, \omega=\nabla \times \mathbf{u}$ and $\mathbf{j}=\nabla \times \mathbf{b}$ )

$$
\begin{aligned}
\frac{\partial \omega}{\partial t} & =\nabla \times\left[\mathbf{u} \times\left(\omega+2 \Omega_{\mathrm{o}}\right)+\mathbf{j} \times\left(\mathbf{b}+\mathbf{B}_{\mathrm{o}}\right)\right]+v \nabla^{2} \omega \\
\frac{\partial \mathbf{b}}{\partial t} & =\nabla \times\left[\mathbf{u} \times\left(\mathbf{b}+\mathbf{B}_{\mathrm{o}}\right)\right]+\eta \nabla^{2} \mathbf{b} .
\end{aligned}
$$

In equations (2) and (3), $\mathbf{u}, \omega, \mathbf{b}$ and $\mathbf{j}$ are the turbulent velocity, vorticity, magnetic induction and electric current density, respectively, of the magneto-fluid.

If we set $v=0$ in (2) and $\eta=0$ in (3), we obtain the equations of ideal MHD turbulence. While any real flows have $v \neq 0$ and $\eta \neq 0$, a study of ideal turbulence

produces some interesting theoretical results that may help in understanding aspects of real turbulence, particularly at larger scales of the flow where dissipation is minimal.

\section{IDEAL MHD TURBULENCE}

Define the volume average of a quantity $\phi(\mathbf{r})$ in a periodic box of side length $2 \pi$ as

$$
[\phi] \equiv(2 \pi)^{-3} \int \phi(\mathbf{r}) d^{3} x
$$


TABLE 1. Invariants for ideal MHD turbulence.

\begin{tabular}{cccc}
\hline Case & Mean Field & Rotation & Invariants \\
\hline I & $\mathbf{B}_{\mathrm{o}}=0$ & $\Omega_{\mathrm{o}}=0$ & $E, H_{C}, H_{M}$ \\
II & $\mathbf{B}_{\mathrm{o}} \neq 0$ & $\Omega_{\mathrm{o}}=0$ & $E, H_{C}$ \\
III & $\mathbf{B}_{\mathrm{o}}=0$ & $\Omega_{\mathrm{o}} \neq 0$ & $E, H_{M}$ \\
IV & $\mathbf{B}_{\mathrm{o}} \neq 0$ & $\Omega_{\mathrm{o}} \times \mathbf{B}_{\mathrm{o}} \neq 0$ & $E$ \\
V & $\mathbf{B}_{\mathrm{o}} \neq 0$ & $\Omega_{\mathrm{o}}=\sigma \mathbf{B}_{\mathrm{o}}$ & $E, H_{P}$ \\
\hline
\end{tabular}

Since all functions are periodic, we can use equations (2) through (4), along with integration by parts to derive the following relations (see [Shebalin 2002]):

$$
\begin{aligned}
\frac{d E}{d t} & =-2(v \Omega+\eta J), \\
\frac{d H_{C}}{d t} & =\Omega_{\mathrm{o}} \cdot[\mathbf{b} \times \mathbf{u}]-\frac{1}{2}(v+\eta)[\mathbf{j} \cdot \omega], \\
\frac{d H_{M}}{d t} & =\mathbf{B}_{\mathrm{o}} \cdot[\mathbf{b} \times \mathbf{u}]-\eta[\mathbf{j} \cdot \mathbf{b}] .
\end{aligned}
$$

Above, we have the (volume-averaged) energy $E$, enstrophy $\Omega$, mean-squared current $J$, cross helicity $H_{K}$ and magnetic helicity $H_{M}$ :

$E=\frac{1}{2}\left[u^{2}+b^{2}\right], \quad \Omega=\frac{1}{2}\left[|\omega|^{2}\right], \quad J=\frac{1}{2}\left[|\mathbf{j}|^{2}\right], \quad H_{C}=\frac{1}{2}[\mathbf{u} \cdot \mathbf{b}], \quad H_{M}=\frac{1}{2}[\mathbf{a} \cdot \mathbf{b}]$.

At this point we note that if $\Omega_{\mathrm{o}}=\sigma \mathbf{B}_{\mathrm{o}}$, i.e., if $\Omega_{\mathrm{o}}$ and $\mathbf{B}_{\mathrm{o}}$ are non-zero and parallel, then eq. (6) can be added to $-\sigma$ times eq. (7) to yield

$$
\frac{d H_{P}}{d t}=-\frac{1}{2}(v+\eta)[\mathbf{j} \cdot \omega]+\sigma \eta[\mathbf{j} \cdot \mathbf{b}], \quad H_{P} \equiv H_{C}-\sigma H_{M} .
$$

The quantity $H_{P}$ will be called the 'parallel helicity.'

When $v=\eta=0$, equations (5), (6), (7) and (9) lead us immediately to the invariant integrals for MHD turbulence for various values of $\mathbf{B}_{\mathrm{o}}$ and $\Omega_{\mathrm{o}}$. These various cases are placed in Table 1 for easier reference. We see that as a mean field is applied or an overall rotation is imposed or both, the number of invariants drop from three to two to one.

The probability distribution function $D$ of the absolute equilibrium ensemble, for Case I in Table 1, has the general form

$$
D \sim \exp \left(-\alpha E-\beta H_{C}-\gamma H_{M}\right) .
$$

The function $D$ is normalized by requiring that its integral over the whole phase space defined by the independent Fourier coefficients be unity. In regard to the other cases in Table 1, eq. (10) applies as follows: Case II, set $\gamma=0$; Case III, set $\beta=0$; Case IV, set $\beta=\gamma=0$; and in Case $\mathrm{V}$, set $\gamma=-\sigma \beta$, so that eq. (10) becomes $D \sim \exp \left(-\alpha E-\beta H_{P}\right)$.

Thus, to determine various ensemble predictions for Cases II through V in Table 1, we merely take previously determined results for Case I [Frisch 1975], [Shebalin 1989, 1994, 2002, 2006], [Stribling 1990] and substitute for $\beta$ and $\gamma$ as indicated above. 

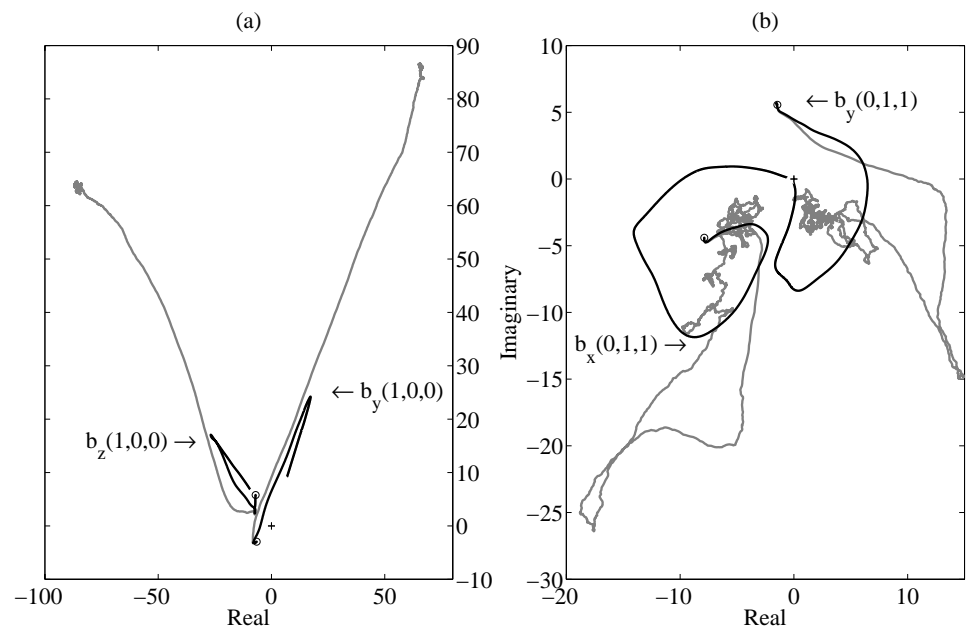

FIGURE 1. Phase portraits for Case I in Table 1. Re vs Im parts of $\tilde{\mathbf{b}}(\mathbf{k})$, where $\mathbf{k} \cdot \tilde{\mathbf{b}}(\mathbf{k})=0$, are plotted for (a) $\mathbf{k}=(1,0,0)$ and (b) $\mathbf{k}=(0,1,1)$, from $t=0$ to 300 . The runs were on a $64^{3}$ grid with $v=\eta=1 / 250$. The initial points $(t=0)$ are indicated by $\circ$. Run 1A results are in gray and those of Run 1 are in black.

Using (10), ensemble predictions for moments of the $\tilde{\mathbf{u}}(\mathbf{k})$ and $\tilde{\mathbf{b}}(\mathbf{k})$ can be made for the different cases in Table 1 . Denoting the ensemble prediction of a quantity $Q(\mathbf{k})$ by $\langle Q(\mathbf{k})\rangle$, the predicted first-order moments are $\langle\tilde{\mathbf{u}}(\mathbf{k})\rangle=\langle\tilde{\mathbf{b}}(\mathbf{k})\rangle=0$ for all cases. The ensemble predictions for second-order moments are not needed here, but are given in [Shebalin 2006] for the five cases listed in Table 1.

An apparent challenge in using eq. (10) is that $H_{C}, H_{M}$ and $H_{P}$ are pseudoscalars under the discrete symmetries of charge inversion $C$ and spatial inversion (or parity) $P$, in which case $D$ in eq. (10) may not be invariant under $C$ and $P$. However, it can be shown that the inverse temperatures $\beta$ and $\gamma$ in eq. (10) are also pseudoscalars under $C$ and $P$ [Shebalin 1996, 1998, 2002, 2006]. Thus, the probability density function indicated by eq. (10) is symmetric under $C$ and $P$, as are the MHD equations (2) and (3).

The basic result is that when eq. (10) is used for ensemble averaging, the integral over phase space includes disjoint components identified by plus and minus signs of the helicity. The number of disjoint components for the cases in Table 1 is $2^{M}$, where $M$ is the number of invariant helicities listed in each of the 5 cases (i.e., Case I has four components, Case IV has one component, and Cases II, III and IV have two components each). This disjoint structure was first pointed out in [Shebalin 1998]; applying the Birkhoff-Khinchin theorem [Khinchin 1949] tells us that only Case IV is ergodic, while Cases I, II, III and V are non-ergodic.

However, when a numerical simulation is run or a physical system evolves, we can start with either the plus or the minus sign of a given helicity, but not both. Thus the symmetry of the theory is dynamically broken, and mean values of the Fourier coefficients with respect to time are not necessarily zero, even though this is the ensemble prediction. Numerical examples of this are presented next. 


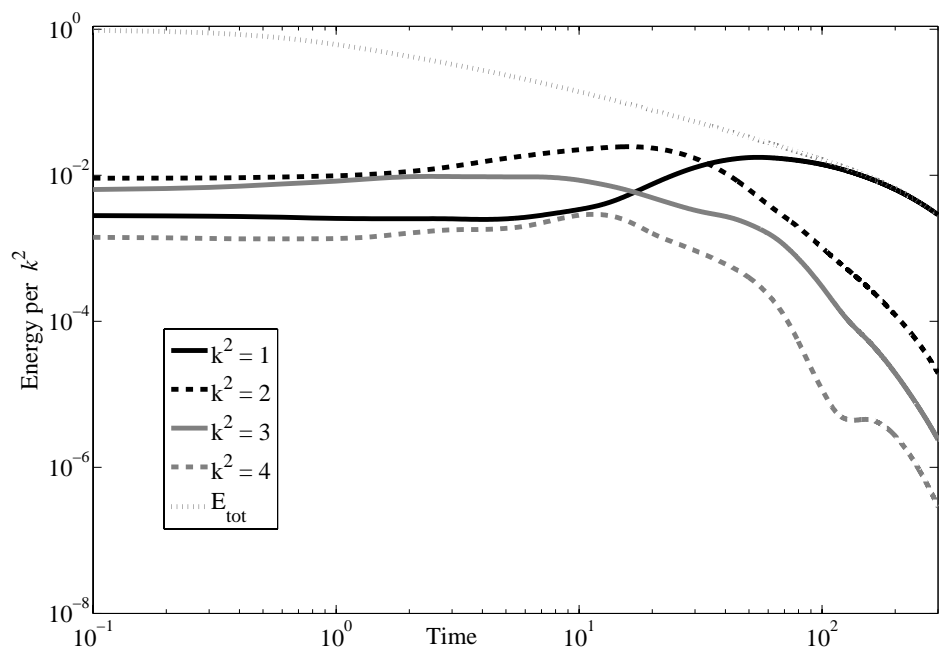

FIGURE 2. Energy in modes with given $k^{2} \leq 4$ vs time. $E_{t o t}$ is the energy in all modes.

\section{REAL \& IDEAL NUMERICAL RESULTS}

A Fourier spectral transform method [Patterson 1971] with $3^{\text {rd }}$-order time-integration [Gadzag 1976] was used for numerical studies. Ergodicity can be examined using phase diagrams, i.e., plots of the real versus the imaginary parts of a Fourier coefficient. Consider the time history of several Fourier modes $\tilde{\mathbf{b}}(\mathbf{k})$ from two $64^{3}$ Case I runs $\left(\mathbf{B}_{\mathrm{o}}=\Omega_{\mathrm{o}}=0, t=0\right.$ to 300$)$ : Run 1A $(v=\eta=0)$ and Run $1(v=\eta=0.004)$; both runs had identical initial conditions: $E(k) \sim k^{4} \exp \left(0.5 k / k_{p}\right), k_{p} \approx 6$, with random phase. Initially, total kinetic and magnetic energies were equal, with $E=1, H_{C}=0.32$ and $H_{M}=0.09$. Phase diagrams are presented comparing the same $\tilde{\mathbf{b}}(\mathbf{k})$ for Runs $1 \mathrm{~A}$ and 1 in Figure 1: (a) $\mathbf{k}=(1,0,0)$ and (b) $\mathbf{k}=(0,1,1)$. In Figure 2, the time evolution of energy in modes with $k^{2}=1,2,3$ and 4 , as well as the total energy for all modes, for real Run 1 is shown.

Although the ensemble prediction is that Fourier modes such as $\tilde{\mathbf{b}}(\mathbf{k})$ are zero-mean random variables, this does not match the time behavior shown in Figure 1, particularly for $k^{2}=1$. Non-ergodicity is clearly evident and is due to the aforementioned broken symmetry. Also note that Figure 1 shows that $\tilde{\mathbf{j}}(\mathbf{k})=i \mathbf{k} \times \tilde{\mathbf{b}}(\mathbf{k}) \sim \tilde{\mathbf{b}}(\mathbf{k})$, which appears to be a general result, indicating 'depression of nonlinearity' [Kraichnan 1988], i.e., tendency to a force-free state where the Lorentz force $\mathbf{j} \times \mathbf{b} \approx 0$. Furthermore, the $k^{2}=1$ modes track each other fairly closely initially; in Figure 1 (a), the ideal trajectory arrives at its attractor at $t \approx 70$, while the real trajectory peaks at $t \approx 52$ (the phase path ends at $t=300)$. The $\tilde{\mathbf{u}}(\mathbf{k})$ for $k^{2}=1$ also show similar behavior.

Next, consider Figure 2, where the transfer of energy between shells of different $k$ is shown. Although the total energy is monotonically decaying, the energy in $k^{2}=4$, 3,2 and 1 modes peaks at $t \approx 2,10,20$ and 50, respectively. This indicates an inverse cascade [Frisch 1975]; coupled with the attractor evident in Figure 1, we have a coherent structure or magnetic dynamo. 


\section{CONCLUSION}

Although the simulations discussed were on a relatively small grid of $64^{3}$ points, this allowed for runs from $t=0$ to 300 (there is always this trade-off). In the ideal Run 1A, broken symmetry and non-ergodicity (i.e., 'broken ergodicity' [Palmer 1982]) leads to coherent structure similar to that found in recent $32^{3}$ runs [Shebalin 2006] that evolved over $t=0$ to 2000 ,. In the real Run 1 , we saw a phase point trajectory close to that of ideal Run $1 \mathrm{~A}$, up until $t \approx 50$. We expect that lower dissipation runs on larger grids will show even longer lived and more energetic magnetic dynamos.

I would like to thank Dr. David Garrison of the University of Houston-Clear Lake for use of the Physics Program computing facility.

\section{REFERENCES}

Elsässer 1956. ～W. M. Elsässer , Hydromagnetic Dynamo Theory, Rev. Mod. Phys., 28 (1956) 135163.

Frisch 1975. U. Frisch, A. Pouquet, J. Leorat, and A. Mazure, Possibility of an inverse cascade of magnetic helicity in magnetohydrodynamic turbulence, J. Fluid Mech., 68 (1975), 769-778.

Gadzag 1976. J. Gadzag, Time-differencing schemes and transform methods, J. Comp. Phys., 20 (1976), 196-207.

Khinchin 1949. $\quad$ A. I. Khinchin, "Mathematical Foundations of Statistical Mechanics," Dover, New York, 1949, 137-145.

Kraichnan 1988. R. H. Kraichnan and R. Panda, Depression of nonlinearity in decaying isotropic turbulence, Phys. Fluids, 31 (1988), 2395-2397.

Lee 1952. T. D. Lee, On some statistical properties of hydrodynamical and magnetohydrodynamical fields, Q. Appl. Math., 10 (1952), 69-74.

Palmer 1982. $\quad$ R. G. Palmer, Broken ergodicity, Adv. Phys., 31 (1982), 669-735.

Patterson 1971. G. S. Patterson and S. A. Orszag, Spectral calculation of isotropic turbulence: Efficient removal of aliasing interaction, Phys. Fluids, 14 (1971), 2538-2541.

Shebalin 1982. J. V. Shebalin, Anisotropy in MHD turbulence due to a mean magnetic field, Ph.D. Dissertation, College of William and Mary, 1982.

Shebalin 1983. J. V. Shebalin, W. H. Matthaeus, and D. Montgomery, Anisotropy in MHD turbulence due to a mean magnetic field, J. Plasma Phys., 29 (1983), 525-47.

Shebalin 1989. J. V. Shebalin, Broken ergodicity and coherent structure in homogeneous turbulence, Physica D, 37 (1989), 173-191.

Shebalin 1994. J. V. Shebalin, Broken symmetry in ideal magnetohydrodynamic turbulence, Phys. Plasmas, 1 (1994), 541-547.

Shebalin 1996. J. V. Shebalin, Absolute equilibrium entropy, J. Plasma Phys., 56 (1996), 419-427.

Shebalin 1998. J. V. Shebalin, Phase space structure in ideal homogeneous turbulence, Phys. Lett. A, 250 (1998), 319-322.

Shebalin 2002. J. V. Shebalin, The statistical mechanics of ideal homogeneous turbulence, NASA TP-2002-210783, Johnson Space Center, Houston, TX, 2002.

Shebalin 2005. J. V. Shebalin, Theory and simulation of real and ideal magnetohydrodynamic turbulence, Discrete \& Continuous Dynamical Systems B, 5 (2005), 153-175.

Shebalin 2006. J. V. Shebalin, Ideal magnetohydrodynamic turbulence in the presence of rotation and a mean magnetic field, J. Plasma Phys., 72 (2006), 507-524.

Stribling 1990. T. Stribling and W. H. Matthaeus, Statistical properties of ideal three-dimensional magnetohydrodynamics, Phys. Fluids B, 2 (1990), 1979-1988.

Woltjer 1958. $\quad$ L. Woltjer, A theorem on force-free magnetic fields, Proc. Nat. Acad. Sci. USA, 44 (1958), 489-491. 